\title{
Effect of normal aging on the manipulation of information in working memory
}

\author{
SYLVIE BELLEVILLE, NANCIE ROULEAU, and NICOLE CAZA \\ Université de Montréal, Montréal, Québec, Canada
}

\begin{abstract}
The goal of this study is to examine the central executive of working memory in normal aging, specifically focusing on its capacities to manipulate or modify the format of the to-be-recalled material. The central executive was measured with the alphabetical span procedure, during which subjects were asked to recall a random series of words in their alphabetical order. The storage demand was equalized across subjects by adjusting the list lengths according to individual span. Experiments 1,2 , and 3 showed that elderly subjects were not impaired in manipulating information, relative to young controls, even when the difficulty of the task was increased. In Experiment 4, validity was tested by asking young subjects to perform the task under the conditions of full or divided attention. Alphabetical recall was more impaired than direct recall during the divided attention condition, which suggests a larger involvement of the central executive component in the former. These results are discussed in relation to the hypothesis of a central executive impairment being associated with normal aging.
\end{abstract}

Working memory (WM) is a short-term memory system involved in tasks that require concurrent retention and processing (Baddeley, 1986; Baddeley \& Hitch, 1974, 1994; Salthouse, 1994). In Baddeley's theoretical framework, WM is a tripartite system composed of two specialized slave systems - the phonological loop and the visuospatial sketchpad-and a central executive. The phonological loop contributes to the retention of verbal information and is subdivided into a passive phonological store and a rehearsal procedure. The visuospatial sketchpad holds images and spatial information. The central executive is an attentional component involved in the control and distribution of limited resources (Baddeley, 1986, 1992; Baddeley \& Hitch, 1974). This study focuses on the manipulation capacities of the central executive in normal aging. Manipulation is defined as the process of actively and consciously modifying the format of the information to be recalled. Within a WM framework, manipulation would require involvement of the central executive component.

This work was supported by a grant from the Medical Research Council of Canada (MRC; PG-28) and from le Fond de la Recherche en Santé du Québec (FRSQ) to S.B., by a chercheur-boursier fellowship from the FRSQ to S.B., and by a scholarship from the FCAR to N.R. and N.C. The authors thank Laurent Mottron, François Crépeau, Geoffrey R. Loftus, Frank Bellezza, and Leah Light for their helpful comments on an earlier version of the text. Thanks to Michel Lamoureux and Jean-François Angers for statistical advice, as well as to Fanny Guérin, Sherif Karama, and Sonia Litalien for testing assistance. Special thanks to Rodolphe Sauvage for conducting Experiment 3. Finally, the authors gratefully acknowledge Ronald Dixon for his comments and for editing the text. Correspondence concerning this article should be addressed to Sylvie Belleville, Centre de Recherche de l'Institut Universitaire de Gériatrie de Montréal, 4565 Queen-Mary, Montréal, Québec, Canada H3W 1W5 (e-mail: bellevis@ere.umontreal.ca).

-Accepted by previous editor, Geoffrey R. Loftus
Recent theoretical and empirical attempts have been made to examine the functioning of the central executive component and to specify the nature of its involvement in cognitive activities. One example is the model for action control elaborated by Norman and Shallice (1986), which was proposed as a potentially fruitful theoretical framework for the central executive. This model suggests that well-known actions are performed through the activation of automatic schemas. When activated schemas are incompatible (e.g., opening a door while holding a bag), semiautomatic plans of action (or contention scheduling, in the Norman and Shallice terminology) occur to resolve the conflict. Hence, the bag would be rapidly transferred to the nondominant hand, allowing use of the dominant hand to open the door. There are situations that cannot be resolved by simple contention scheduling, and in these cases the system can rely on a second form of control: the supervisory attentional system (SAS). This form of control is nonautomatic; it is slow and conscious. The SAS acts by modulating the pattern of activation of particular schemas so that the most appropriate actions take place. For example, the SAS would be involved in a situation in which a subject has to open a door while holding a bag in one hand and a cup of coffee in the other hand. In this case, the subject might stop, look around for a nearby place to rest the coffee, put down the cup of coffee, open the door, and subsequently pick up the cup of coffee. In the Baddeley and Hitch view (Baddeley, 1986, 1992; Baddeley \& Hitch, 1994), the central executive of WM corresponds to the SAS. According to this framework, the central executive would be involved in a number of different activities: realization of new actions, planning and decision making, time sharing, inhibition of automatic behavior, as well as the updating and manipulation of information in WM.

A number of studies have observed that elderly people are impaired in tasks that require the manipulation of the 
information retained in WM (Craik, 1986; Dobbs \& Rule, 1989; Foos, 1989; Tun, Wingfield, \& Stine, 1991; Wright, 1981). One interesting example of a manipulation task is provided by Craik (1986). Gick and Craik (reported in Craik, 1986) assessed manipulation abilities in normal aging with an alphabetical span procedure. The subjects were presented a random series of words, which they were required to rearrange mentally and to report in alphabetical order. Serial ordered recall is usually spontaneously adopted by subjects during the immediate recall of short lists. One reason might be that the format of recall is then perfectly congruent with the format of presentation. Furthermore, the phonological loop retains an ordered representation of the material. In contrast, alphabetical recall requires that subjects organize and control their retrieval. This corresponds closely to one of the roles that the SAS system is hypothesized to play in memory. When asked to modify the format of the input, the subject has to break down the ordered representation, extract alphabetical order from long-term memory, and scan that order while items are held in the phonological loop. The subject has to verify the output while controlling these different operations. Completion of this task is impossible with automatic processes and very unlikely with simple contention scheduling. Rather, the task requires that automatic schemas (for example, reading the content of the ordered representation in the phonological loop) be inhibited and that information from long-term memory be activated. The alternation between the reading of each information type-the items and the alphabet-would then be done by modulation of each process. The task most likely requires usage of the SAS control system, or central executive, the role of which is to provide control over cognitive operations. The performances of elderly and young normals on the alphabetical recall of words and on serial digit recall were compared by Gick and Craik. Elderlies were found to be impaired on the alphabetical task, yet were unimpaired relative to young normals on the digit span task. The authors suggested that the alphabetical recall impairment was not due to a decrease in passive storage, since aged and young subjects did not differ when reporting sequences of digits. This led them to conclude that aged subjects were impaired in WM tasks involving active manipulation of the material.

Although the original alphabetical span procedure is quite appealing, it suffers from a potentially important methodological weakness. Specifically, the use of digit span might not have been an appropriate control for passive storage capacities. The implicit assumption in the protocol used by Gick and Craik (Craik, 1986) is that only one difference exists between alphabetical and digit recall: the first task requires manipulation, whereas the second does not. It must be noted, however, that the two conditions also differ in their material format: words versus digits. This difference in material format may underlie the apparent interaction between age and recall type. Stud- ies that have compared digit versus word spans have at times observed intact digit spans but impaired word spans in normal aging (Light \& Anderson, 1985, Experiment 1; Wingfield, Stine, Lahar, \& Aberdeen, 1988). In these studies, a difference of about one unit was found between the word spans of young subjects and those of aged subjects. This corresponds to the difference in the alphabetical recall of words, reported by Craik, between the two age groups. Consequently, the age difference observed by Gick and Craik in the alphabetical condition may simply reflect a word span impairment rather than an impairment in manipulating information. This word span reduction is also indicative of an impaired storage capacity in aged subjects, a suggestion that is congruent with other studies (Dobbs \& Rule, 1989; Foos, 1989). A passive storage deficit in elderly people might thus be responsible for their observed difficulties in manipulating information, as it makes them particularly sensitive to tasks requiring both the retention and the manipulation of information.

Loftus (1978) has pointed out the complexities of interpreting interaction effects in memory research, because the shape of the function that relates response probability to a particular theoretical component is generally unspecified. Salthouse (1985) has raised the same concerns in aging research, mentioning that the frequently observed baseline differences between young and elderly subjects is a serious problem when examining interaction effects. The finding of an interaction between the variable of interest and age is obscured if the two groups differ according to their baseline performance, as this variable of interest may have differential effects when it occurs at different points of the performance range. For example, manipulation operations are certainly more difficult to perform on weak memory traces than on strong ones. The direct implication is that a performance deficit may be manifested, not because manipulation is impaired, but rather because the operation acts on a weaker trace. Other problems may arise when the two groups have different baselines. For example, a ceiling effect in young subjects but not in older ones might be responsible for the detection of an interaction. One solution proposed to resolve the problem of unequal baselines is to manipulate the conditions of testing to ensure comparable levels of performance in both groups (Belleville, Malenfant, Peretz, \& Chatelois, 1992; Meudell \& Mayes, 1982; Salthouse, 1985; Salthouse, Fristoe, Lineweaver, \& Coon, 1995; Somberg \& Salthouse, 1982).

The alphabetical span procedure represents an attractive tool for the assessment of manipulation abilities; we thus reemployed this procedure to assess manipulation abilities in aged individuals. However, the present study controlled for an impairment in the storage capacities of older subjects before introducing a manipulation requirement into the task. This was done by first assessing each subject's word span and by adjusting the length of the experimental sequences according to this span. The sub- 
jects were then tested with word series in the condition of direct (or serial) recall and in the condition of alphabetical recall. Because of the adjustment of the length of the sequences relative to each subject's span, the performance of aged and young subjects should not differ in the direct recall condition. However, if aged subjects experience a genuine manipulation deficit, they should be impaired in the alphabetical condition. In Experiment 1, the subjects were tested with sequence lengths that were one word smaller than their individual span capacity. In Experiments 2 and 3, the number of items to manipulate in the task was increased by one, in order to make the task more demanding.

Experiment 4 addressed the construct validity of the different procedures used to assess the central executive of WM. Construct validity refers to a task's ability to be a true measure of the component that it is proposed to examine. Independent evidence that particular tasks or dependent variables are true measures of central executive function represent crucial validation, especially in experiments failing to report age differences. Even if there are theoretical reasons for believing that alphabetical recall involves the SAS system, it is still possible that the task can be performed with semiautomatized forms of controls. In order to address this problem, we took advantage of the fact that time-sharing paradigms call on the central executive. A third experiment assessed whether the alphabetical span is sensitive to divided attention. Young healthy subjects were asked to perform the direct and alphabetical recall tasks in either a divided attention condition or a full attention condition. According to Baddeley (1996), the central executive is involved in the division of attention or time sharing. Performing a task that requires extensive central executive capacities (alphabetical recall) should be particularly problematic when the central executive is simultaneously involved in dualtask coordination. Divided attention is likely to alter performance on any concurrent task, provided it is nonautomatic. However, if alphabetical recall requires more central executive input than does direct recall, it should also be more susceptible to divided attention.

\section{EXPERIMENT 1}

In this experiment, young and elderly normal subjects were required to report a sequence of words in the presented order or in alphabetical order. The subjects were tested with individually adjusted sequences, the length of which corresponded to their word span minus one item. The immediate serial recall procedure used in the direct condition was the same as that used in a typical span test. Since the sequence length was adjusted according to individual word spans, the performance of young versus elderly subjects should not differ on the direct recall condition. However, if the manipulation capacities of the central executive are dysfunctional in aged subjects, they should be impaired in the alphabetized condition, relative to young subjects.

\section{Method}

\section{Subjects}

Thirty-two normal francophone subjects, 16 young and 16 elderly, participated in the experiment. All subjects were taken from a pool of volunteers living in the community. They had no history of neurological disease, psychiatric disorder, or recent (within a year) general anesthesia and did not take any medications known to affect cognitive functions. A medical questionnaire was used to assess the inclusion criteria prior to the subjects' acceptance into the protocol. The young subjects ( 6 males and 10 females) were, on average, 22.38 years of age $(S D=2.63$, range $19-28)$ and had a mean of 13.31 years of education $(S D=1.54)$. The older subjects ( 2 males and 14 females) had a mean age of 71.75 years $(S D=4.67$, range $68-78)$ and a mean educational level of 12.75 years $(S D=3.51)$. The difference in formal education between aged and young subjects was not significant $(0.56 \pm 1.97$, where 1.97 is the $95 \%$ confidence interval). General verbal performance was assessed with the Mill Hill Vocabulary Test (Gérard, 1983). This is a widely used francophone test in which subjects have to identify the synonym of a target word from among six choices. On this test, young subjects had a mean score of $32.15 / 44$ ( $S D=4.81$; the results for 1 young subject were not available on this test), and older subjects had a mean score of $35.56 / 44(S D=5.48)$. The difference in vocabulary score was not significant $(3.41 \pm 3.76)$.

\section{Materials}

Two hundred and eleven monosyllabic words were chosen according to the following criteria: (1) the words had to be frequent (Baudot, 1992) and imaginable substantives, and (2) the words were unambiguous with respect to the print-to-sound correspondence of their first letter. For example, a word like phare in French (which is pronounced [fAR]) would have been excluded. Furthermore, words having homophones of a higher frequency were not used.

These words were used to construct sequences of words, the length of which ranged from two to eight items (to be used with subjects of a span size from three to nine). There were 20 different sequences for each length, 10 to be used in the direct condition and 10 to be used in the alphabetical condition. Words included in a sequence differed according to their first letter and had no phonological or semantic similarity. In addition, the manipulation requirement was controlled by measuring three factors: (1) the distance between the first letter of the words in a sequence (according to a pilot study from our laboratory [unpublished data], it is easier to judge the alphabetical order of letters that are farther apart); (2) their position in the alphabet, since letters at the beginning of the alphabet should be easier to arrange in alphabetical order; and (3) the total number of manipulations it was necessary to perform in each sequence (e.g., there is one manipulation in the series bloc, pneu, and crabe but two manipulations in the series rose, pomme, and lune). With these criteria, the manipulation requirement was made equivalent for the different sequence lengths. Mean word frequency was equivalent across sequence length, as well as for the direct and alphabetical condition at a given list length. None of the words were repeated across different sequences for a given length. Sequences of different lengths (thus submitted to subjects of different span sizes) originated from the same pool of words but were not identical with respect to order and items, for the reasons outlined below. For example, Subject A, with a span size of seven, was tested with 20 sequences of six items ( 10 in alphabetical and 10 in direct). The 20 sequences were different and made up of nonrepeated words. Subject $B$, with a span of six, was also tested with 20 sequences. These 
were necessarily different from those used for Subject $A$, as they contained one less item. However, sequences were controlled so that frequency and manipulation requirements were equivalent for both subjects. We did not construct the different sequence lengths by simply removing (or adding) one item from the longer (or smaller lists), because it was impossible to do so without causing serious imbalance across sequences in the manipulation difficulty.

\section{Procedure}

Preexperimental phase. As a first step, a classical word span procedure was used to assess the short-term memory capacity of each subject. The words chosen for the span procedure fit the same criteria as those on the experimental list, in terms of length, frequency, and imageability, yet did not overlap with the experimental list. The items were chosen without replacement. Sequences of words were read to the subjects at the rate of one item per sec, starting with short sequences of two words. The length of the sequences was increased by one word every two trials. However, if an error occurred on one of these two trials, the subjects were given two additional trials. The subjects were instructed to orally report items in serial order. Testing was interrupted when the subjects failed to report correctly two of the four sequences at a particular length. The word span was defined as the longest sequence correctly recalled on $50 \%$ of the trials.

Experimental phase. Following the span measurement, the subjects were assessed in two conditions-direct and alphabetical recall. Words were read to the subjects at the rate of one item per sec, and the subjects recalled the words orally. In the direct condition, the subjects performed an immediate serial recall of the words. In the alphabetical condition, they were asked to rearrange and recall the words in their alphabetical order. For example, the words routenappe-poivre should be recalled nappe, poivre, route in this condition. Ten sequences of words were recalled in each condition. The number of words to be recalled in a sequence corresponded to the subject's span, as determined in the preexperimental phase, minus one item. For example, a subject with a word span of five was tested with sequences of four words. The order of presentation of the direct and alphabetical conditions followed an ABBA design, starting with the direct condition. This allowed us to control for possible effects of fatigue or practice. The whole procedure was completed within a single testing session.

\section{Results and Discussion}

A preliminary analysis was performed that compared the span sizes of young and aged subjects as assessed in the preexperimental phase. The average span was smaller in aged subjects $(M=4.38, S D=0.72)$ than in young subjects $(M=4.88, S D=0.81)$, and this difference was marginally significant $(0.5 \pm 0.547)$. A second preliminary analysis was done to assess the effect of the order of presentation because of the ABBA design. A 2 (age: young, old) $\times 2$ (order: first, second) $\times 2$ (recall: direct, alphabetical) analysis of variance (ANOVA) was conducted. The analysis showed that neither the main effect of order nor any of the interactions with this factor reached significance $(F<1$, in all cases). This indicates that the order of presentation of the conditions had no influence on performance and that no fatigue or practice effects were affecting the results. For this reason, the data from the two orders of presentation were pooled in the subsequent analyses.

Figure 1 shows the number of sequences correctly recalled by elderly and young subjects in both conditions. To be considered as correct, the whole sequence of items

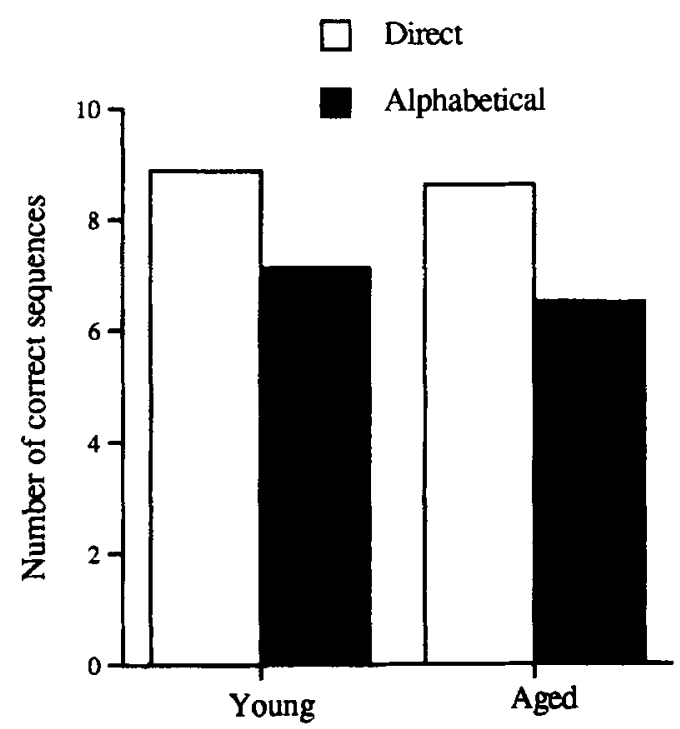

Figure 1. Number of sequences correctly recalled by young and aged subjects in conditions of direct (white columns) and alphabetical recall (shaded columns) of Experiment 1. Subjects were tested using sequences with lengths that were one item smaller than their span.

had to be correctly recalled. Alphabetical recall yielded a diminished absolute level of performance, in comparison to direct recall (see Figure 1). However, the decrement that was due to alphabetical recall is not larger in older subjects. This is confirmed by a 2 (age: young, old) $\times 2$ (recall: direct, alphabetical) ANOVA. The analysis revealed a significant main effect of recall $[F(1,30)=$ $45.76, M S_{\mathrm{e}}=1.31, p<.001$, reflecting the reduced performance level in the alphabetical condition. A power analysis indicated that the effect size of the main effect of recall (Hintze, 1991, according to Cohen, 1988) was 0.846 . There was no main effect of age $\left(F<1, M S_{\mathrm{e}}=\right.$ 4.53 ), indicating that elderly and young subjects exhibited the same performance level. The effect size for the main effect of age was 0.145 , and a group of 388 subjects would be required in order to reach a power level of .80 . Contrary to the prediction, the age $\times$ recall interaction was not significant $(F<1)$. A power analysis of the interaction showed an effect size of only 0.03 and indicated that a sample size of 630 subjects would be required in order to reach a power level of .80 .

Another set of ANOVAs was performed using the proportion of correctly recalled items (see Table 1). Since the number of items represents a finer measure of recall, it might be more sensitive to an interaction between age and condition. An item was considered as correct when it was recalled in the position appropriate to the recall condition (serial or alphabetical). The total number of words recalled was then transformed into a percent correct value. The same ANOVA as above was performed and showed similar insignificant results (no interaction, $F<1$ ).

In summary, alphabetical recall yields a decreased performance level in comparison to direct recall, which sug- 
Table 1

Proportion of Correct Items (With Standard Deviations) for Young and Aged Subjects Tested at Span Minus One

\begin{tabular}{cccccc}
\hline & \multicolumn{3}{c}{ Condition } \\
\cline { 2 - 3 } \cline { 5 - 5 } Subjects & \multicolumn{2}{c}{ Direct Recall } & & \multicolumn{2}{c}{ Alphabetical Recall } \\
\cline { 2 - 3 } \cline { 5 - 6 } & $P($ Corr. $)$ & $S D$ & & $P($ Corr $)$ & $S D$ \\
\hline Young & 96.44 & 4.07 & 84.60 & 12.32 \\
Aged & 93.90 & 8.63 & & 81.31 & 11.80 \\
\hline
\end{tabular}

gests that there is a cost to the manipulation requirement of the task. However, elderly and young subjects, equalized in the direct condition, do not perform differently in the alphabetical condition. This suggests that manipulation is not particularly difficult for normal elderly people, as long as basic storage is controlled for. The absence of an age effect in alphabetical recall may, however, relate to a relative lack of task sensitivity. It must be noted that all of the subjects performed quite well on both the direct and alphabetical conditions; this might be explained by the observation that the subjects were tested below their span limits. In fact, a ceiling effect was observed in most of the subjects in the direct condition, as well as in some of the subjects in the alphabetical condition. Another possibility is that the task was not sufficiently demanding and, thus, that the proposed deficit in elderly normals would be manifested by a more rigorous condition that tests the limits of the SAS. For this reason, a second experiment was devised, in which the task was made more difficult by increasing the number of items in each sequence by one.

\section{EXPERIMENT 2}

In this experiment, the procedure was slightly modified in order to eliminate performance at the ceiling level and to increase the task demand. This was done by increasing the length of the sequences by one item. The subjects were thus tested at the very limit of their shortterm memory capacity with sequence lengths corresponding to their span.

\section{Method}

Subjects

Thirty-two different subjects ( 16 young normals and 16 elderly normals) participated in this experiment. The subjects were selected according to exclusion and inclusion criteria that were the same as those in Experiment 1 . The young subjects $(7$ males and 9 females) were, on average, 22.31 years of age ( $S D=2.73$, range 19-26) and had completed, on average, 14.25 years of education $(S D=1.84)$. The older subjects ( 2 males and 14 females) had a mean age of 73.69 years $(S D=3.55$, range 66-79) and a mean educational level of 11.69 years $(S D=3.01)$. The difference in formal education between aged and young subjects was significant $(2.56 \pm 1.82)$, with the young normals having achieved slightly higher educational levels. However, the general verbal performance, as measured with the Mill Hill Vocabulary Test, was significantly higher in aged subjects $(M=38.21, S D=3.38$ ) than in young subjects $(M=34.62, S D=4.56$; note that 2 young subjects did not complete the task), $3.59 \pm 2.92$.
}

\section{Materials \\ The materials were the same as those in Experiment 1.}

\section{Procedure}

The procedure was the same as that in Experiment 1, except that the number of words to be recalled corresponded to each subject's span size, as determined in the preexperimental phase.

\section{Results and Discussion}

Two preliminary analyses were performed. First, the span sizes of young and elderly subjects were compared. Elderly subjects showed a smaller span than young subjects ( 4.7 words in young and 4.4 in elderly), and this difference was marginally significant $(0.30 \pm 0.354)$. The effect of the presentation order of the conditions was again assessed with a 2 (age: young, elderly) $\times 2$ (order: first, second) $\times 2$ (recall: direct, alphabetical) ANOVA. There was no main effect of order of presentation $(F<1)$, and none of the interactions with order reached significance [age $\times$ order and recall $\times$ order, $F<1$; age $\times$ order $\times$ recall, $\left.F(1,30)=1.67, M S_{\mathrm{e}}=0.92, p=.21\right]$. The data from the two orders were thus pooled in the following analyses.

Figure 2 shows the number of sequences recalled correctly in both the direct and the alphabetical conditions. As in Experiment 1, performance in the alphabetical condition is severely depressed, as compared to that in the direct order condition. However, this reduction is again equivalent for both groups. This was confirmed by an ANOVA that revealed a significant main effect of recall $\left[F(1,30)=42.46, M S_{\mathrm{e}}=2.80, p<.001\right]$, associated with an effect size of 1.15 , but no interaction between age and recall $(F<1)$. A power analysis of the interaction yielded an effect size of 0.169 and indicated that the sample size required here to reach a power level of .80 would be 288 subjects. Note that, according to Figure 2,

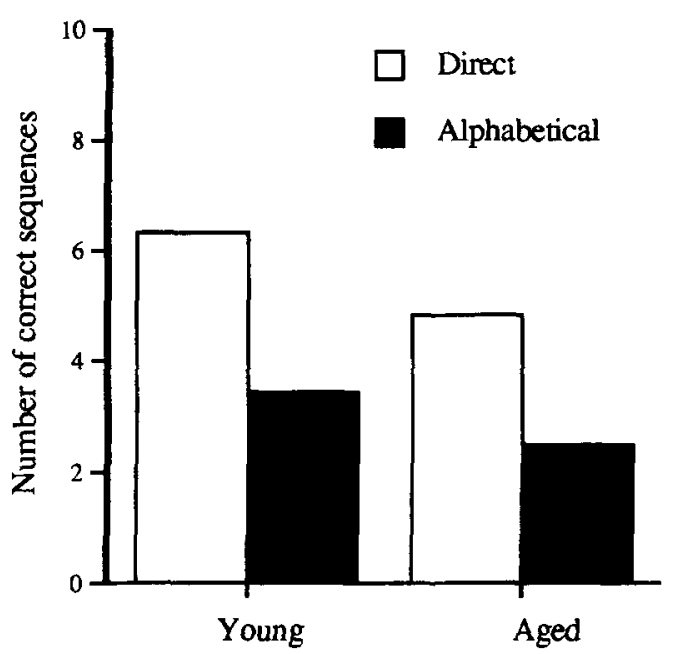

Figure 2. Number of sequences correctly recalled by young and aged subjects in conditions of direct (white columns) and alphabetical recall (shaded columns) of Experiment 2. Subjects were tested with sequences corresponding to their span. 
Table 2

Proportion of Correct Items (With Standard Deviations) for Young and Aged Subjects Tested at Span in Experiment 2 and Experiment 3

\begin{tabular}{|c|c|c|c|c|}
\hline \multirow[b]{3}{*}{ Subjects } & \multicolumn{4}{|c|}{ Condition } \\
\hline & \multicolumn{2}{|c|}{ Direct Recall } & \multicolumn{2}{|c|}{ Alphabetical Recall } \\
\hline & $P$ (Corr.) & $S D$ & $P$ (Corr) & $S D$ \\
\hline \multicolumn{5}{|c|}{ Experiment 2} \\
\hline Young & 85.38 & 9.15 & 64.19 & 15.02 \\
\hline Aged & 73.55 & 18.42 & 53.13 & 15.76 \\
\hline \multicolumn{5}{|c|}{ Experiment 3} \\
\hline Young & 93.56 & 6.98 & 75.20 & 8.85 \\
\hline Aged & 90.56 & 8.66 & 73.50 & 17.42 \\
\hline
\end{tabular}

the interaction would be in the opposite direction from that expected - that is, the age difference would be larger in the direct than in the alphabetical condition. Although aged subjects do appear to have a lower global level of performance, the main effect of age was not significant $\left[F(1,30)=2.26, M S_{\mathrm{e}}=8.35, p=.14\right]$. The effect size of the main effect of age was 0.2657 , and 114 subjects would be required to reach a power level of 80 .

As in Experiment 1, an analysis was conducted using the percentage of correctly recalled items as the dependent variable (see Table 2). The results of the analysis were similar to those obtained when correctly recalled sequences was used as the dependent variable: the interaction was not significant $(F<1)$, yet the main effect of age was significant $\left[F(1,30)=5.95, M S_{\mathrm{e}}=352.03\right.$, $p<.05]$.

In conclusion, elderly subjects do not appear to show any differential effects of alphabetical recall on WM, when compared to young subjects. The procedure used here intended to equalize recall in the direct condition by testing subjects at their short-term memory span. Unfortunately, we have only partially succeeded in doing so. Although young and aged subjects recall an equivalent number of sequences, this is not the case when measuring the number of correct items. Young subjects do recall more items than do older ones in the direct condition. It is worth pointing out that there is clearly no interaction between age and condition, in spite of this age effect on the number of items: the age difference is not larger in the alphabetical condition than in the direct condition. However, the presence of a group effect is a problem, because it is still possible that an interaction would come out in a transformed version of the data (Loftus, 1978). In principle, this group effect should not have been found, because the subjects were tested with sequence lengths that corresponded to their spans as measured in the preexperimental phase. One possible explanation is that aged subjects are simply more sensitive to fatigue. Although possible, this explanation is disconfirmed by the fact that no group $\times$ order effect was found in the preliminary analyses on the experimental trials. However, fatigue effects could have occurred between the preexperimental and experimental trials. As a result, it is possible that the span measurement was not accurate in aged subjects. We have evidence to suggest that this might be the case in aged subjects and that they were outperforming their baselines during the span measurement procedure of the preexperimental phase. In the preexperimental phase, the span measurement is done with only 2 trials at each length. The subjects move rapidly through the incremental list lengths, as only 2 trials are used to estimate the span. If the number of trials is not sufficient to take into account a potential fatigue effect in the aged, these subjects may fail to maintain the same level of performance in the experimental phase, where 10 trials are given in each condition. An examination of the data suggests that this is the case. First, the span size measured in the preexperimental phase is quite large for our aged subjects and does not differ statistically from that for younger ones. Second, Figure 2 shows that aged subjects recall less than $50 \%$ of the sequences in the direct condition ( $48.3 \%$ precisely). Yet, the sequences presented should yield a higher performance level, as it is supposed to correspond to their span, which is defined as the length for which subjects reach a minimum of $50 \%$ correct performance. On the few trials available in the preexperimental phase for the same lengths, the performance of aged subjects was much higher, reaching about $70 \%$. There was thus a decrease in performance from the preexperimental to the experimental trials. Of note, a look at individual data shows that it is those subjects tested with the fewest trials that show the largest decrease from the preexperimental to the experimental phase. The measure used here thus seems to have overestimated the span size of some of our aged subjects, because not enough trials were provided at each length. This, interacting with fatigue effects, contributed to an alteration of the span estimate for aged subjects. A third experiment was undertaken to take this into account with regard to span measure, in an attempt to eliminate the group effect.

\section{EXPERIMENT 3}

In Experiment 3, the span measurement procedure in the preexperimental phase was slightly modified in order to provide a more accurate estimate of span capacity, because we suspected that errors in the span measurement of our elderly subjects were the source of our failure to equalize performances in Experiment 2. The procedure was thus modified to allow a more rigorous criterion for span capacity. This should ensure equalization of recall across groups in the direct condition and allow us to measure interactions without the possible confounding effect of differences in baseline performance.

\section{Method}

\section{Subjects}

Twenty new subjects ( 10 young and 10 elderly) participated in this experiment. The subjects were selected according to exclusion and inclusion criteria that were the same as those in the previous experiments. The young subjects ( 10 females) were, on average, 21.70 
years of age ( $S D=0.95$, range 20-23) and had completed, on average, 16.40 years of education $(S D=1.07)$. The older subjects ( 1 male and 9 females) had a mean age of 73 years $(S D=4.55$, range $68-79)$ and a mean educational level of 15.50 years $(S D=1.51)$. The difference in formal education between aged and young subjects was not significant $(0.90 \pm 1.07)$. However, the general verbal performance, as measured with the Mill Hill Vocabulary Test, was significantly higher in aged subjects $(M=29.40, S D=2.88)$ than in young subjects $(M=26, S D=3.92 ; 3.40 \pm 0.82)$.

\section{Materials}

The materials were the same as those in Experiment 1

\section{Procedure}

The procedure was the same as that in Experiment 2, except for the span measurement of the preexperimental phase, which differed as explained below. Span was established with a staircase procedure. One trial was presented for each sequence length, starting with sequences of two items. A correct recall was followed by the presentation of sequences one item longer. When subjects failed on a trial, 3 more trials of the same sequence length were presented. If subjects succeeded on 2 trials, 6 other trials were provided, for a total of 10 trials. If the subjects did not recall 2 of the trials, a total of 10 trials were presented at the smaller sequence length. A $50 \%$ correct performance on a series of 10 trials was required for the sequence length to be considered as the subject's span. A 20-min pause was provided to the subjects between the preexperimental phase and the experimental phase.

\section{Results and Discussion}

As a preliminary analysis, the span sizes of young and elderly subjects were compared. Elderly subjects showed a smaller span than did young subjects ( 4.9 words in the young and 4.2 in the elderly; $0.7 \pm 0.31$ ). Figure 3 shows the number of sequences recalled correctly in both the direct and the alphabetical conditions. The ANOVA revealed a significant main effect of recall $[F(1,18)=49.23$,

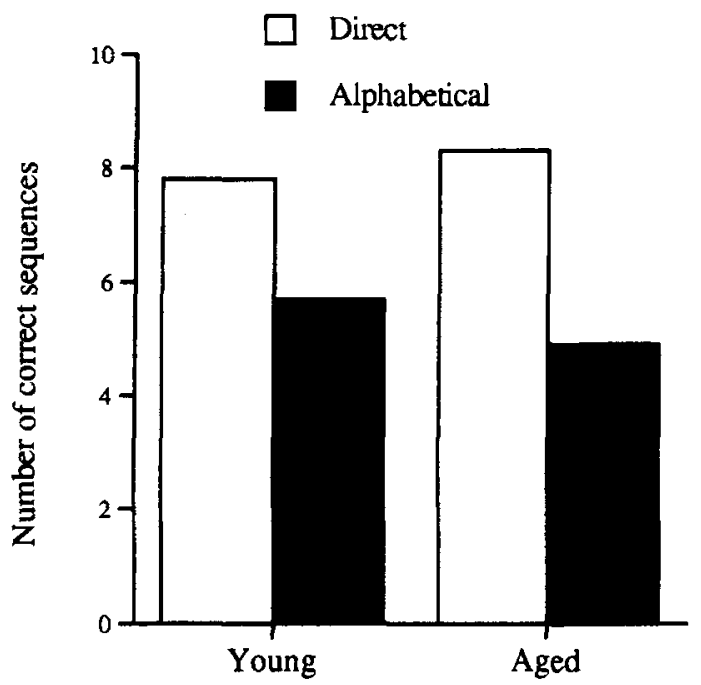

Figure 3. Number of sequences correctly recalled by young and aged subjects in conditions of direct (white columns) and alphabetical recall (shaded columns) of Experiment 3. Subjects were tested with sequences corresponding to their span.
$\left.M S_{\mathrm{e}}=75.63, p<.001\right]$ but neither an age effect $(F<1)$ nor an interaction between age and recall $[F(1,18)=$ $\left.2.75, M S_{\mathrm{e}}=4.23, p=.11\right]$. A power analysis of the interaction showed an effect size of only 0.1943 and indicated that a sample of 192 subjects would be required in order to reach a power level of .80 . The same results were obtained when the percentage of correctly recalled items was used as the dependent variable (see Table 2): neither the main effect of age nor the interaction was significant $(F<1$ in both cases), yet the main effect of recall was $\left[F(1,18)=38.05, M S_{\mathrm{e}}=26.1, p<.001\right]$. The power analysis was done for the interaction and showed a somewhat larger effect size (0.3909). It also indicated that the use of 50 subjects would yield a power of 80 . However, it is worth pointing out here that the interaction would be in the direction opposite to that predicted by an age-related manipulation decline (see Table 2).

In summary, a modification of our span measurement technique was sufficient to permit an equalization of the performance of aged and young subjects in the direct recall condition. Yet again, no interaction between group and recall was observed, further confirming that elderly subjects are not more impaired by the alphabetical recall condition, when compared with young subjects.

\section{EXPERIMENT 4}

The goal of Experiment 4 was to evaluate the validity of our manipulation procedure. For that purpose, the same paradigm was applied to young subjects in the condition of either full or divided attention. In the divided attention condition, the subjects performed either the direct or the alphabetical recall condition, as well as a concurrent visuospatial tracking task. This concurrent task was chosen because it does not compete with words for space in the verbal short-term store or for phonological processing. Thus, any performance decline in the divided attention condition can be explained by central executive involvement in coordinating the two tasks. If alphabetical recall requires the central executive, it should thus be more affected than is direct recall in the divided attention condition.

\section{Method}

Subjects

In all, 32 French-speaking students volunteered to participate in the experiment. The inclusion criteria were the same as those for Experiments 1,2 , and 3 . The subjects were randomly assigned to the two different conditions of attention-full or divided. The first group (full attention) was composed of 7 men and 9 women between the ages of 19 and $30(M=21.63, S D=3.52)$. Their average level of education was 14.84 years $(S D=2.71)$, and their average score on the Mill Hill Vocabulary Test was 34.50 ( $S D=$ 2.85 ). The second group (divided attention) included 6 men and 10 women between the ages of 18 and $29(M=22.06, S D=3.13)$. This group had a mean of 14.87 years of education $(S D=2.10)$ and an average score on the Mill Hill Vocabulary Test of 36.75 ( $S D=$ $2.95)$. No significant differences in age $(0.43 \pm 2.42)$ or formal education $(0.03 \pm 1.76)$ were found. Scores on the Mill Hill Test just reached significance $(2.25 \pm 2.11)$. 


\section{Materials and Apparatus}

This experiment utilized word span and memory tasks identical to those described for Experiments 1 and 2. In addition, a visuospatial tracking task was required for the divided attention condition. This latter task was presented on the screen of a Macintosh Classic computer and consisted of maintaining, via the computer mouse, a rectangular cursor $(0.2 \times 0.5 \mathrm{~cm})$ within a diagonally moving rectangle $(0.7 \times 0.9 \mathrm{~cm})$.

\section{Procedure}

Preexperimental phase. The subjects were tested individually. The word span was measured for all of the subjects by using a procedure that was the same as that for Experiments 1 and 2. Then the tracking threshold on the visuospatial task was established. This started with a familiarization phase, during which the subjects were introduced to computer mouse manipulation and to the tracking task. During this phase, the subjects tracked the moving target once at slow paces $(0.2 \mathrm{pixel} / \mathrm{sec}$ and $0.4 \mathrm{pixel} / \mathrm{sec})$ along a horizontal, vertical, and oblique axis. Then the tracking threshold was obtained for each subject. During 10-sec trials, the subjects were required to track the oblique moving target. The speed of the target changed on every trial. The initial speed was set at $0.5 \mathrm{pixel} / \mathrm{sec}$ and was increased by $0.2-\mathrm{pixel} / \mathrm{sec}$ increments after each trial. Increments were continued until subjects failed to maintain the cursor in the target during $65 \%$ of the trial, as recorded by the computerized program. Speed was then decreased by $0.2 \mathrm{pixel} / \mathrm{sec}$ on every trial until the subject reached $90 \%$ correct tracking performance. The presentation of such ascending and descending series of speeds was repeated until a $70 \%$ correct performance level (between $65 \%$ and $75 \%$ ) was obtained for a given speed on two successive trials. Therefore, for each subject, a specific target speed was obtained that corresponded to the fastest speed at which he or she maintained performance approximately $70 \%$ of the time. This represented the speed that was to be used in the experimental phase. All of the subjects were required to complete the tracking threshold measure, in order to ensure that the two groups did not differ in regards to fatigue.

Experimental phase. After the tracking threshold measure, the subjects underwent the memory task according to a procedure that was the same as that in Experiments 1 and 2 . In addition, the subjects in the divided attention condition were asked to perform the visuospatial tracking task concurrently with the presentation and recall of the word sequences. The target in the tracking task moved obliquely at threshold speed. The subjects were instructed to maintain tracking accuracy at the same level as that measured in the preexperimental phase. They were thus required to put emphasis on the visuospatial task. These instructions were given in order to reduce tradeoff effects with the memory task. Thus, an average performance of about $70 \%$ correct tracking was expected on the visuospatial tracking task, whether performed concurrently with either the direct or the alphabetical condition. A tracking accuracy measure was taken after every five sequences, in agreement with the ABBA order of presentation.

\section{Results}

The average word spans for each group were compared. The subjects in the full attention condition had a mean span of 5.06 words $(S D=0.77)$, and the subjects in the divided attention condition had a mean span of 4.94 words $(S D=0.68)$. The difference in span between the two groups was not significant $(0.12 \pm 0.53)$. The effect of presentation order was then assessed with a 2 (attention: full, divided) $\times 2$ (order: first, second) $\times 2$ (recall: direct, alphabetical) ANOVA. No main effect of order was found $[F(1,30)=1.55, p=.2233]$, and this

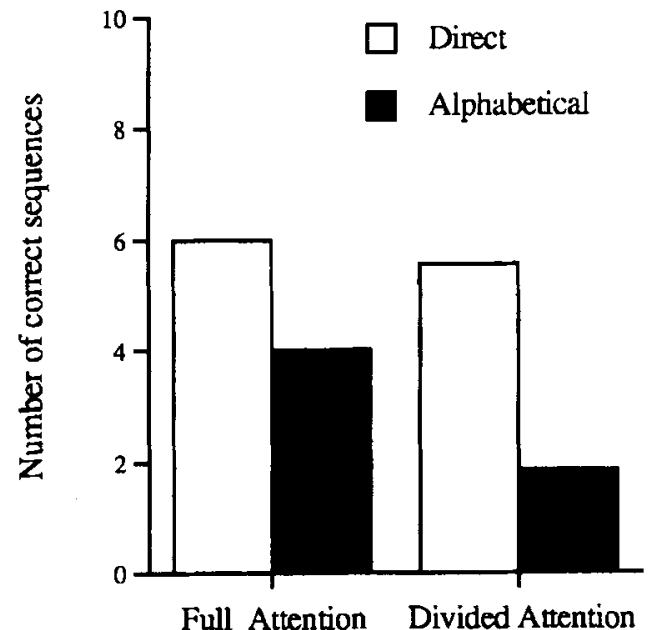

Figure 4. Performance of young subjects in the direct (white columns) and alphabetical (shaded columns) recall conditions when tested in the condition of full or divided attention.

factor did not interact with attention $[F(1,30)=1.55$, $p=.22]$. However, the order $\times$ recall interaction was significant $[F(1,30)=5.24, p<.05]$. Simple effects analysis showed an order effect in the direct condition $[F(1,30)=$ $\left.11.68, M S_{\mathrm{e}}=0.39, p<.01\right]$. Performance on the last five trials was superior to performance on the first five trials, a result that is congruent with a practice effect. However, there was no order effect in the alphabetical condition $(F<1)$. The absence of a three-way interaction is of note $(F<1)$. Consequently, the results obtained from the different orders were pooled in the following analyses.

Figure 4 presents the direct and alphabetical recall of the sequences for each attention group. Examination of this figure indicates that the performance decline in the alphabetical condition is larger for the subjects in the concurrent divided attention condition than for those in the full attention condition. The ANOVA performed on the number of correct sequences yielded a significant main effect of recall $\left[F(1,30)=60.92, M S_{\mathrm{e}}=2.12, p<\right.$ .0001 ; effect size, 1.38]. This confirms that fewer sequences were recalled in alphabetical order than in direct order. The main effect of attention was marginally significant $\left[F(1,30)=3.40, M S_{\mathrm{e}}=7.73, p=.0752\right]$, with an effect size of 0.33 . However, main effects were qualified by a significant interaction between recall and attention $\left[F(1,30)=5.36, M S_{\mathrm{e}}=2.12, p<.05\right]$, with an effect size of 0.41 . An analysis of the simple effects showed that no attention effect was present in the direct condition $(F<1)$, but that this effect was present in the alphabetical condition $\left[F(1,30)=8.10, M S_{c}=4.46, p<.005\right]$. Furthermore, the reduction that was due to alphabetical recall was larger in the divided attention condition $[F(1,30)=$ $\left.51.22, M S_{\mathrm{e}}=2.12, p<.0001\right]$ than in the full attention condition $\left[F(1,30)=15.07, M S_{\mathrm{e}}=2.12, p<.001\right]$. Subsidiary analyses using the percentage of items recalled (see Table 3) confirmed the analysis performed on the 
Table 3

Proportion of Correct Items (With Standard Deviations) of Focused and Divided Attention

\begin{tabular}{lcrccc}
\hline & \multicolumn{4}{c}{ Condition } \\
\cline { 2 - 3 } \cline { 5 - 6 } Subjects & \multicolumn{2}{c}{ Direct Recall } & & \multicolumn{2}{c}{ Alphabetical Recall } \\
\cline { 2 - 3 } \cline { 5 - 6 } Focused attention & $P$ Corr. $)$ & $S D$ & & $P$ Corr. $)$ & $S D$ \\
\hline Divided attention & 79.87 & 9.15 & & 69.49 & 13.82 \\
\hline
\end{tabular}

number of correct sequences, as described above: the interaction was statistically significant $[F(1,30)=4.27$, $\left.M S_{\mathrm{e}}=100.80, p<.05\right]$.

We also assessed performance on the tracking task in order to verify whether the interaction effect relates to the presence of a tradeoff. When recalling the words in direct order, the subjects showed an average tracking performance of $70.01 \%(S D=8.33$, range $54-82)$, whereas, when recalling words in alphabetical order, tracking performance was at $68.07 \%(S D=8.67$, range $54.4-84.4)$. Tracking performance in both conditions was fairly close to the tracking threshold ( $70 \%$ in principle), and the difference across conditions was not significant ( $1.94 \pm 2.18$, two-tailed). There was thus no evidence of a tradeoff between the two tasks.

\section{GENERAL DISCUSSION}

The results of our four experiments are quite straightforward. First, the alphabetical span procedure, which requires the manipulation of information within WM, is more difficult than a passive task in which direct recall is required. Furthermore, alphabetical span is more affected by divided attention. This is congruent with the substantial role of the central executive in the performance of the manipulation task. Surprisingly, elderly normal subjects' performance on the manipulation tasks is identical to that of young controls. These manipulation abilities of elderly people are retained when the difficulty of the manipulation task is increased.

Before discussing the theoretical relevance of these data, we shall first assess whether any of the methodological aspects of our task can explain the pattern of results observed here. The questions of power, sensitivity of the dependent variable, and relevance of the measure will be addressed. We will also discuss how the equalization procedure might account for the difference between our data and those previously reported in the literature.

First, it is possible that our failure to observe a difference between the two age groups was related to insufficient power. This is particularly important in analyses in which the null hypothesis is confirmed. For this reason, we performed power analyses on our data, which showed a very small effect size for the interaction between recall and age. Furthermore, the number of subjects required to obtain a power of 0.80 was above 250 in both Experiments 1 and 2 . When such a large number of subjects is required for a significant difference to be obtained, the effects are usually judged as negligible (Cohen, 1988).
When a smaller number of subjects was required (as in Experiment 3), the interaction found was in the direction opposite to that which is expected in the case of a manipulation defect. It is important to note that the significant effect of recall (direct compared with alphabetical recall) yielded large effect sizes (Cohen, 1988), indicating that the manipulation procedure was sound and afforded little variability. This result was replicated with different samples in three experiments. Although the problem of lack of power is an important issue in aging research, we do not believe that it is sufficient to explain these results.

An alternative explanation is that the absence of an age effect might relate to a lack of sensitivity in the measure. More precisely, it is possible that the manipulation requirement of the alphabetical condition was insufficient to identify a difference between the two groups. This criticism cannot be completely rejected, yet there are three reasons that lead us to believe that our procedure was sensitive to a central executive deficit. First, the alphabetical recall condition was clearly more demanding than was the direct recall condition, because it systematically yielded a lower performance level. Furthermore, this effect was large and robust. Second, we showed in Experiment 4 that a small resource reduction in young subjects was sufficient to decrease their performance substantially on the alphabetical recall condition, relative to direct recall. Finally, Experiments 2 and 3 added to the task demand by potentially increasing the number of manipulations to be performed in WM. It is of note that the performance decline in the alphabetical condition, relative to the direct condition, was larger in the second and third experiments than in the first experiment. This suggests that the task was indeed more difficult for the subjects and thus taxed the limits of the system. Nonetheless, aged subjects remained unimpaired in the alphabetical recall condition, relative to young subjects. We are thus quite confident that a lack of sensitivity is insufficient to explain the absence of an interaction between age and recall.

Another possible objection regarding our results is that the task used here is not a true measure of central executive functioning. However, both theoretical and empirical arguments suggest that the alphabetical task is a genuine measure of the central executive component of WM. Manipulation tasks require that subjects organize and control their retrieval strategies, and this fits one of the functions occupied by the central executive. Nonautomatized, complex, or new tasks would involve conscious control from the central executive (or the SAS; Norman \& Shallice, 1986). This control would take place by modulating existing schemas. This theoretical argument was confirmed in Experiment 4 , which showed that alphabetical recall was more sensitive to the concurrent completion of a tracking task than was direct recall. The utilization of a concurrent visuospatial task precluded competition for space in a phonological loop. Rather, the coordination of the two tasks probably required the control of the central executive system. The fact that divided attention dramatically di- 
minished alphabetical recall, especially when compared with its effects on direct recall, supports the notion that the task requires commitment from the central executive.

The observation of normal manipulation capacities in aging is in disagreement with a number of previous studies. Other experiments have reported adult age differences on tasks that have also involved a manipulation of the information retained in WM (Craik, 1986; Dobbs \& Rule, 1989; Foos, 1989; Tun et al., 1991; Wright, 1981). This was thought to reflect the impairment experienced by aged individuals in novel and demanding situations, or in conditions requiring active processes, and was explained by a central executive defect. One of these previous experiments used the alphabetical span procedure presented here, but with digits as the control condition (Craik, 1986). Although statistical details are not provided by the author, the results indicate that elderly people are impaired on an alphabetical recall condition but not on a typical digit span task (Craik, 1986).

One major difference between our paradigm and those used in the past is that we have equalized storage requirements across groups, and this control procedure might be sufficient to account for the different outcomes. Indeed, studies that have assessed either processing capacities or storage capacities have found impairments in elderly subjects (Dobbs \& Rules, 1989; Foos, 1989; Light \& Anderson, 1985; Wingfield et al., 1988). In the present study, a small but systematic difference was observed between the two groups' word spans. Thus, the performances of elderly normal subjects and of young normal subjects are not completely akin; aged subjects appear to have a smaller storage capacity. If we had tested both groups with sequences of equal lengths, therefore, the task would have been slightly easier for young subjects than for elderly subjects. Furthermore, a reduction in storage capacity makes older subjects more vulnerable to a task that requires a manipulation of the information in addition to storage. The lack of control for storage differences in past studies may have induced a spurious manipulation decrement. Aged subjects were compared with young subjects in conditions that were more difficult for them for reasons other than the manipulation requirement. Our control procedure ensured that a storage impairment did not contribute to the manipulation deficit of elderly individuals.

One might object that the equalization procedure modified the nature of the task. It may be argued that testing subjects with sequences differing in size qualitatively modifies the task across the two groups of subjects. However, the equalization procedure was not arbitrarily chosen: we ensured that subjects were tested at the limit of their short-term memory capacity by using a classical estimate of this capacity (i.e., the span task). The number of items to recall thus corresponded to a genuine psychological limit for each individual subject. Consequently, we believe that equalizing subjects ensured that the task was qualitatively equivalent across subjects. When passive storage was controlled for, as in the present case, the manipulation deficit of aged individuals vanished.
A similar pattern has occurred in time-sharing tasks. Impaired levels of performance have been observed in elderly subjects on divided attention paradigms (Inglis \& Caird, 1963; McDowd \& Craik, 1988; Salthouse, Rogan, \& Prill, 1984; Tun et al., 1991; Wright, 1981). However, many studies have also failed to observe age differences in divided attention (e.g., Baddeley, Logie, Bressi, Della Sala, \& Spinnler, 1986; Belleville, Malenfant, et al., 1992; Gick, Craik, \& Morris, 1988; Morris, Gick, \& Craik, 1988; Somberg \& Salthouse, 1982; Wickens, Braune, \& Stokes, 1987). The discrepancy observed here might relate to the failure of previously used paradigms to control for the processing requirement of the individual tasks. The time-sharing deficit observed on occasion in elderly subjects may thus have arisen from a difficulty in performing the individual tasks, and this difficulty would be exacerbated in a condition in which they had to perform both tasks simultaneously (Belleville, Malenfant, et al., 1992; Hartley, 1992; Salthouse et al., 1995). Elderly individuals are thus probably not more impaired than young people on time-sharing paradigms, as long as the degree of difficulty of the individual tasks is made comparable for the two groups.

The absence of a manipulation deficit when controlling for passive storage capacities is of theoretical relevance for the hypotheses regarding the source of the agerelated decline in cognition. Salthouse $(1988,1991)$ has questioned the level at which age-related impairments are found and has proposed that normal aging only affects elementary processes. In his view, a restricted number of general elementary processes (or resources) underlie the functioning of the specialized cognitive components. An impairment of one of these elementary processes in normal aging would thus affect a large number of cognitive activities to varying degrees. In normal aging, complex cognitive tasks would be difficult to perform only because they rely on impaired elementary processes. These elementary processes remain to be identified, but possible candidates have been proposed by Salthouse. These included working memory, speed, and attention. Both the storage and the manipulation aspects of working memory were foreseen as impaired elementary processes (Salthouse, 1991). However the present experiment indicates that the manipulation aspect of working memory is not a primary source of the age-related impairment. In contrast, the present results are compatible with storage capacities being a primary source of the age-related impairment. Indeed, it can be argued that the equalization procedure had the effect of compensating for a deficiency at the level of one of the elementary processes. Given that the equalization of storage capacities was performed, our results would suggest that storage space might be one of the elementary resources impaired in aging. Other investigators have proposed that reduced storage is a primary source of impairment in normal aged people. For example, Parkinson and collaborators (Inman \& Parkinson, 1983; Parkinson, Inman, \& Dannenbaum, 1985) have observed that matching subjects on the basis of storage space eliminates many of the age-related memory impairments. 
Yet this storage impairment is probably not related to a phonological deficit or to an absence of rehearsal procedures. There have been numerous descriptions of patients who, after localized brain damage, present with a verbal working memory deficit that is related to an impairment of phonological storage (see, e.g., Vallar \& Baddeley, 1984) or of articulatory rehearsal (see, e.g., Belleville, Peretz, \& Arguin, 1992; Waters, Rochon, \& Caplan, 1992). These patients are characterized by a severe span reduction, resulting in a capacity of $2-3$ items, and are also not sensitive to the phonological similarity and/or articulatory length of the items. The pattern of impairment in normal aged individuals is different, inasmuch as their span reduction is much smaller and we have shown them to be as sensitive as normal young subjects to the phonological similarity and length of items in immediate serial recall (Belleville, Peretz, \& Malenfant, 1996). Nonetheless, it remains possible that articulatory speed or storage space is reduced in normal aging.

It is important to point out that age differences have been reported in central executive tasks other than manipulation and time-sharing paradigms. For example, aged individuals are impaired when the syntactic complexity of the to-be-verified sentences on the sentence span procedure is increased (Gick et al., 1988; Morris et al., 1988; Wright, 1981). Although this has been interpreted as arising from a deficit in the active process of working memory, it may also relate to a specific impairment in syntactic processing. Van der Linden and collaborators (Van der Linden, Brédart, \& Beerten, 1994) have reported age differences in a task that requires subjects to monitor and update information in WM. Baddeley (1986) has found age differences in a random generation task in which subjects are required to produce series of elements that mimic randomization. This task would require individuals to monitor the ongoing productions and to avoid the repetition of patterns, as well as the production of automatic series or acronyms (such as USA). These paradigms, however, have not controlled for group differences in storage or speed, and the results may be partially accounted for by a deficiency at this level. Alternatively, these tasks might tap into some component process of the central executive that, although different from the component processes investigated here, is affected by normal aging.

Along the same line, there have been numerous recent works showing that aged people are impaired in tasks that are sensitive to frontal lobe damage. For example, aged individuals show deficits in the self-ordered pointing task (Daigneault \& Braun, 1993; Shimamura \& Jurica, 1994) and in more classical tests of frontal executive functions, such as the Stroop or the Wisconsin card-sorting task (WCST; for reviews, see Moscovitch \& Winocur, 1992; West, 1996). They also exhibit impairment in retaining contextual information in long-term episodic memory. Some authors have argued that this pattern of impairment relates to a frontal lobe dysfunction. This frontal lobe hypothesis is congruent with the suggestion that normal aging is accompanied by a deficit in the ex- ecutive component of working memory. However, care must be taken in interpreting previous data on frontal lobe tasks. For example, Houx, Jolles, and Vreeling (1993) have observed that, when considering mild biological or environmental factors, the age effect was much reduced on the Stroop interference test. Paolo and collaborators (Paolo, Tröster, Axelrod, \& Koller, 1995) have shown that the WCST for normal elderly individuals does not strongly share construct validity with other frontal and memory tasks. They suggest that this task provides more information about elderly individuals' problem-solving strategies than about their attentional functions.

Finally, it is worth mentioning that classical tests of frontal lobe functions are not exempt from the methodological limitations reported here for working memory tasks. It is possible that a portion of this frontal lobe deficit would vanish, if appropriate controls of some as yet unknown general factor were performed for these tasks. Nevertheless, it has been shown that, even when controlling for group effects, sume frontal lobe functions remain impaired in normal aging. Parkin and Walter (1992) have shown that an impairment in contextual memory was still present in a comparison of subgroups of young and aged subjects matched with respect to their memory for facts. However, this finding was reported for a task that measured the contribution of frontal lobe functioning to longterm memory and not to working memory. This might again highlight the importance of subdividing the general class of executive functions into finer categories. Executive functions have been measured with a wide range of heterogeneous tasks that do not have the same cognitive requirements and that are likely to reflect a variety of processes. For instance, some of these tasks might assess inhibition (e.g., the Stroop test), whereas others appear to rely largely on problem solving (e.g., the WCST). If there are different executive functions, it is likely that not all are impaired in aged individuals, as is shown here.

In summary, we have failed to observe age differences in a task that requires the active manipulation of information within working memory. This was found in three different experiments and thus appears to be a robust observation. Furthermore, this was observed even when the manipulation demand of the task was increased. The absence of an age decrement could relate to the fact that this study controlled for a potential age-related impairment in basic storage capacity. Thus, diminution in storage capacity might represent a source for the cognitive decline observed in normal aging. The present results cast some doubts on the hypothesis of a general central executive deficit in normal aging. Like time sharing, manipulation is probably not impaired in normal elderly subjects. The central executive of normal aged people is, therefore, at most only partially impaired. However, it is the goal of future research to determine which central executive functions are impaired and which are not and to integrate these empirical findings into a coherent description of the central executive. 


\section{REFERENCES}

BADDELEY, A. [D.] (1986). Working memory. Oxford: Oxford University Press, Clarendon Press.

BADDELEY, A. [D.] (1992). Working memory. Science, 255, 556-559.

BADDELEY, A. [D.] (1996). Exploring the central executive. Quarterly Journal of Experimental Psychology, 49A, 5-28.

Baddeley, A. D., \& Hitch, G. J. (1974). Working memory. In G. H Bower (Ed.), The psychology of learning and motivation (Vol. 8 , pp. 47-90). New York: Academic Press

BADDELEY, A. D., \& Hitch, G. J. (1994). Developments in the concept of working memory. Neuropsychology, 8, 485-493.

Baddeley, A. [D.], Logie, R. [H.], Bressi, S., Della Sala, S., \& SPINNLER, H. (1986). Dementia and working memory. Quarterly Journal of Experimental Psychology, 38A, 603-618.

BAUDOT, J. (1992). Fréquence d'utilisation des mots en français écrit contemporain [Frequency of use of words in contemporary written French]. Montréal: Presses de l'Université de Montréal.

Belleville, S., Malenfant, D., Peretz, I., \& Chatelois, J. (1992, January). The attentional component of working memory in norma aging. Paper presented at the International Congress of Psychology, Brussels, Belgium.

Belleville, S., Peretz, I., \& Arguin, M. (1992). Contribution of articulatory rehearsal to short-term memory: Evidence from a case of selective disruption. Brain \& Language, 43, 713-746.

Belleville, S., Peretz, I., \& Malenfant, D. (1996). Examination of the working memory components in normal aging and in dementia of the Alzeheimer type. Neuropsychologia, 34, 195-207.

COHEN, J. (1988). Statistical power analysis for the behavioral sciences (2nd ed.). Hillsdale, NJ: Eribaum.

CrAIK, F. I. M. (1986). A functional account of age differences in memory. In F. Klix \& H. Hagendorf (Eds.), Human memory and cognitive capabilities: Mechanisms and performances (pp. 409-422). Amsterdam: Elsevier, North-Holland

Daigneault, S., \& Braun, C. M. J. (1993). Working memory and the self-ordered pointing task: Further evidence of early prefrontal decline in normal aging. Journal of Clinical \& Experimental Neuropsychology, $15,881-895$.

DoBbS, A. R., \& RuLE, B. G. (1989). Adults' age differences in working memory. Psychology \& Aging, 4, 500-503.

Foos, P. W. (1989). Adult age differences in working memory. Psy chology \& Aging, 4, 269-275.

GÉRARD, M. (1983). Contribution à l'évaluation de la détérioration mentale chez l'adulte à l'aide du test de vocabulaire Mill Hill [Contribution of the assessment of mental deterioration in the adult with the Mill Hill vocabulary test]. Unpublished master's thesis, University of Liège.

Gick, M. L., CraIK, F. I. M., \& MorRIS, R. G. (1988). Task complexity and age differences in working memory. Memory \& Cognition, 16, 353-361.

Hartley, A. A. (1992). Attention. In F. I. M. Craik \& T. A. Salthouse (Eds.), The handbook of aging and cognition (pp. 3-49). Hillsdale, $\mathrm{NJ}$ : Erlbaum.

Houx, P. J., Jolles, J., \& VReELING, F. W. (1993). Stroop interference: Aging effects assessed with the Stroop color-word test. Experimental Aging Research, 19, 209-224.

Inglis, J., \& CAIRD, W. K. (1963). Age differences in successive responses to simultaneous stimulation. Canadian Journal of Psychiatry, 17, 98-105.

InMAN, V. W., \& Parkinson, S. R. (1983). Differences in BrownPeterson recall as a function of age and retention interval. Journal of Gerontology, 38, 58-64.

Light, L. L., \& ANDERSON, P. A. (1985). Working-memory capacity, age, and memory for discourse. Journal of Gerontology, 40, 737-747.

LoFTUs, G. R. (1978). On interpretation of interactions. Memory \& Cognition, 6, 312-319.

MCDOWD, J. M., \& CRAIK, F. I. M. (1988). Effects of aging and task difficulty on divided attention performance. Journal of Experimental Psychology, 14, 267-280.
Meudell, P., \& Mayes, A. (1982). Normal and abnormal forgetting: Some comments on the human amnesic syndrome. In A. W. Ellis (Ed.), Normality and pathology in cognitive functions (pp. 203-237). London: Academic Press.

Morris, R. G., GiCK, M. L., \& Craik, F. I. M. (1988). Processing resources and age differences in working memory. Memory \& Cognition, 16, 362-366

Moscovitch, M., \& WINOCUR, G. (1992). The neuropsychology of memory and aging. In F. I. M. Craik \& T. A. Salthouse (Eds.), The handbook of aging and cognition (pp. 315-372). Hillsdale, NJ: Erlbaum.

Norman, D. A., \& Shallice, T. (1986). Attention to action: Willed and automatic control of behavior. In R. J. Davidson, G. E. Schwartz, \& D. Shapiro (Eds.), Consciousness and self-regulation (Vol. 4, pp. 1-18). New York: Plenum.

Paolo, A. M., Tröster, A. I., Axelrod, B. N., \& Koller, W. C. (1995). Construct validity of the WCST in normal elderly and persons with Parkinson's disease. Archives of Clinical Neuropsychology, 10, 463-473.

PARKIN, A. J., \& Walter, B. M. (1992). Recollective experience, normal aging and frontal dysfunction. Psychology \& Aging, 7, 290-298.

Parkinson, S. R., Inman, V. W., \& Dannenbaum, S. E. (1985). Adult age differences in short-term forgetting. Acta Psychologica, 60, 83-101.

Salthouse, T. A. (1985). A theory of cognitive aging. New York: Springer-Verlag.

SALTHOUSE, T. A. (1988), Initiating the formalization of theories of $\mathrm{cog}$ nitive aging. Psychology \& Aging, 3, 3-16.

SALTHOUSE, T. A. (1991). Theoretical perspectives on cognitive aging. Hove, U.K.: Erlbaum.

Salthouse, T. A. (1994). Age-related differences in basic cognitive processes: Implications for work. Experimental Aging Research, 20, 249-255.

Salthouse, T. A., Fristoe, N. M., Lineweaver, T. T., \& Coon, V. E. (1995). Aging of attention: Does the ability to divide decline? Memory \& Cognition, 23, 59-71.

Salthouse, T. A., Rogan, J. D., \& Prill, K. A. (1984). Division of attention: Age differences on a visually presented memory task. Memory \& Cognition, 12, 613-620.

Shimamura, A. P., \& JuRica, P. J. (1994). Memory interference effects and aging: Findings from a test of frontal lobe function. Neuropsychology, 8, 408-412.

SOMBERG, B. L., \& SAlthouse, T. A. (1982). Divided attention abilities in young and old adults. Journal of Experimental Psychology: Human Perception \& Performance, 8, 651-663.

Tun, P. A., WingField, A., \& Stine, E. A. L. (1991). Speech-processing capacity in young and older adults: A dual-task study. Psychology \& Aging, 6, 3-9.

VALLAR, G., \& BADDELEY, A. D. (1984). Fractionation of working memory: Neuropsychological evidence for a phonological short-term store. Journal of Verbal Learning \& Verbal Behavior, 23, 151-161.

VAN DER LINDEN, M., BRÉDART, S., \& BEERTEN, A. (1994). Age-related differences in updating working memory. British Journal of Psychology, 85, 145-152.

WaTERs, G. S., Rochon, E., \& CaPLAN, D. (1992). The role of high-level speech planning in rehearsal: Evidence from patients with apraxia of speech. Journal of Memory \& Language, 31, 54-73.

WeST, R. L. (1996). An application of prefrontal cortex function theory to cognitive aging. Psychological Bulletin, 120, 272-292.

Wickens, C. D., Braune, R., \& Stokes, A. (1987). Age differences in the speed and capacity of information processing: 1. A dual-task approach. Psychology \& Aging, 2, 70-78.

Wingfield, A., Stine, E. A. L., Lahar, C. J., \& Aberdeen, J. S. (1988). Does the capacity of working memory change with age? Experimental Aging Research, 14, 103-107.

WRIGHT, R. E. (1981). Aging, divided attention, and processing capacity. Journal of Gerontology, 36, 605-614.

(Manuscript received November 3, 1995; revision accepted for publication February 14, 1997.) 